\title{
Extension of the analytic nodal diffusion solver ANDES to triangular-Z geometry and coupling with COBRA-IIIC for hexagonal core analysis
}

\author{
Juan-Andrés Lozano * Javier Jiménez, Nuria García-Herranz, José-María Aragonés \\ Departamento de Ingeniería Nuclear, Universidad Politécnica de Madrid (UPM), José G. Abascal, 2, 28006 Madrid, Spain
}

A R T I C L E I N F O

\section{Introduction}

During the last years, the reactor physics group at UPM has been working in the development of the in-house nodal neutron diffusion code ANDES (Lozano et al., 2007, 2008a). It is based on the ACMFD method developed by Y.A. Chao in 1999 (Chao, 1999), and extended recently to 3D Cartesian geometry and multigroups (Aragonés et al., 2007). The code has been successfully applied to LWR cores, with square fuel assemblies. ANDES was also coupled with the thermal-hydraulics (TH) code COBRA-IIIC/MIT-2 (Jackson and Todreas, 1981) allowing analysis of LWR at coarse mesh scale also for transient problems (Lozano et al., 2008b), (Jiménez et al., 2008).

The ACMFD method in Cartesian geometry is based on two steps aimed to obtain an analytical solution of the multigroup diffusion equation within homogeneous nodes. The first one is the decoupling of the multigroup diffusion equation by means of diagonalization of the multigroup diffusion matrix. This way we obtain a set of uncoupled diffusion equations over the modal fluxes which are linearly related to the physical fluxes. The second step consists in performing a transverse integration, to reduce the n-dimensional diffusion equation into $\mathrm{n}$ one-dimensional equations, coupled through the transverse leakage included as an external source term.

The key feature of the ACMFD method is that it leads to a linear relation among the node-average fluxes and the interface integrated fluxes and currents at any node face. Such relation is scalar only in the modal space of the eigenvectors associated to each eigenvalue of the multigroup matrix, becoming matrix-vector relations in the physical space of the group fluxes and currents, resulting in the "coupling" of all groups. This leads to a high order scheme since it includes the effects of the intranodal flux shape and spectral variation.

Because of the excellent performance of the ACMFD method for the case of square fuel assemblies, now the objective is its extension to reactor cores with hexagonal fuel assemblies following the same guidelines.

Different nodal codes have been developed for hexagonal geometry applications, including the so mentioned ACMFD method (Chao, 1999). Some of them, such as the NEM (Ivanov et al., 2006), use a transverse integration procedure over the hexagonal 
nodes taking advantage of the superior calculation efficiency of the transverse-integrated nodal methods. However, when applied to a hexagon, the transverse-integrated nodal diffusion equation contains non-physical singular terms, as is explained in (Lawrence, 1986) and (Wagner, 1989); then, some kind of approximation has to be introduced. These approximations significantly degrade the accuracy of the method.

Consequently, most of the nodal codes avoid performing transverse integration over the hexagon. However, the applied methods are not exempt of difficulties either. The method implemented in codes such as ANC-H (Chao and Shatilla, 1995), PANTHER (Knight et al., 1995) or MGRAC (Tomasevic and Müller, 2009), is based on conformal mapping (Chao and Tsoulfanidis, 1995) in order to transform the hexagonal 3D problem into a Cartesian 3D problem before applying transverse integration. In this technique, the mapping scale function modifies the resulting $1 \mathrm{D}$ transverse-integrated equation, making difficult to obtain the analytic flux profile. In the AFEN method (Cho and Noh, 1995) the multigroup diffusion equation is solved directly, by expanding the flux distributions within a node in nonseparable analytic basis functions satisfying the diffusion equations at any point of the node. However, in this approach, the number of continuity conditions (and thus the number of unknowns) at the nodal boundaries needed to obtain the required accuracy, increases the computing time, specially in 3D hexagonal geometry. A variation of the previous method is the HEXagonal Nodal Expansion Method (HEXNEM2) implemented in the DYN3D code (Grundmann and Hollstein, 1999). It is based on a twodimensional expansion of the intranodal fluxes in the hexagon using both 2nd order polynomials and exponential functions. Side-averaged and corner-point values of fluxes and partial currents are used for the coupling of nodes.

Other methods like the TPEN implemented in PARCS (Downar et al., 2004) divide the hexagonal domain into six triangles; for every triangle, the radial flux is represented by using a $2 \mathrm{D}$ polynomial expansion and forcing the solution to fulfill the necessary conditions. This way they avoid transverse integration as in AFEN. However, the continuity conditions to be satisfied among the six triangles require to solve a complex linear system for each hexagonal node, greatly increasing the complexity of the calculation.

In this paper, we demonstrate that the ACMFD method, involving transverse integration, can be satisfactorily extended to reactor cores with hexagonal fuel assemblies when dividing the hexagons into six triangular nodes. The extension to triangular- $Z$ geometry does not involve a substantial change in the procedure used for Cartesian geometry and consequently, the nodal equations remain essentially identical to those for rectangular nodes.

There are several advantages in using triangular nodes: first, it eliminates the appearance of non-physical singular terms in the transverse leakage, reducing the complexity of the transverse-integrated equations; second, it allows to refine the mesh any number of times (with hexagonal nodes it is not possible to subdivide the mesh); and, third, it allows to have a better modelization of the TH and burn-up effects.

The details of the developed formulation, paying special attention to the transverse integration of the diffusion equation in triangular geometry will be summarized in Section 2 . The method has been implemented in the code ANDES, allowing VVER core analyses. The existing TH coupling has also been extended to hexagonal geometry with the capability to model the core using either assembly-wise channels (hexagonal mesh) or a higher refinement with six channels per fuel assembly (triangular mesh). The new coupling scheme and its peculiarities will be explained in Section 3. In Section 4, we verify the performance of the ANDES/COBRA-IIIC coupled system with the steady state cases specified in the V1000CT2-EXT2 Benchmark (Kolev et al., 2007a) and the Exercise 2 of the OECD/ NEA VVER-1000 Coolant Transient Benchmark (Ivanov et al.,
2004). The results corresponding to the transient calculation are provided and the influence of the mesh refinement is analysed.

\section{The analytic coarse mesh finite difference method in triangular-Z geometry}

Let us divide the hexagonal assemblies into six triangular right prisms, composed of five interfaces (two equilateral triangular bases and three rectangular sides).

As explained in the previous section, the first step to obtain the ACMFD relation at each nodal interface is the decoupling of the multigroup diffusion equation; this procedure is independent on the geometry, so all details and notation explained in (Aragonés et al., 2007; Lozano et al., 2008a) are valid. However the second step, that is, the transverse integration process to reduce the $3 \mathrm{D}$ diffusion equation to three $1 \mathrm{D}$ diffusion equations, requires additional developments. For triangular geometry, we will show that the expressions are more complex and further manipulation of the equations is needed.

\subsection{Transverse integration of the $3 D$ diffusion equation in triangular- prismatic nodes}

Let us start from the 3D neutron diffusion modal equation within our homogeneous triangular-prismatic nodal volume $V$ shown in Fig. 1:

$\nabla^{2} \psi_{m}(\vec{r})-\lambda_{m} \psi_{m}(\vec{r})=-S_{m}(\vec{r}) ; \quad m=1, L, G$

where $\lambda_{m}$ is the modal eigenvalue, $\psi_{m}(\vec{r})$ the modal flux, $s_{m}(r)$ the modal external source and $G$ the number of energy groups.

The transverse integration procedure in the axial direction $(z)$ is used to obtain the ACMFD relation at the top and bottom interfaces of the node, in the same way as in Cartesian geometry. However, let us see the form of the resulting equations when applying the transverse integration approach in the radial directions $(x$ or $y$ ).

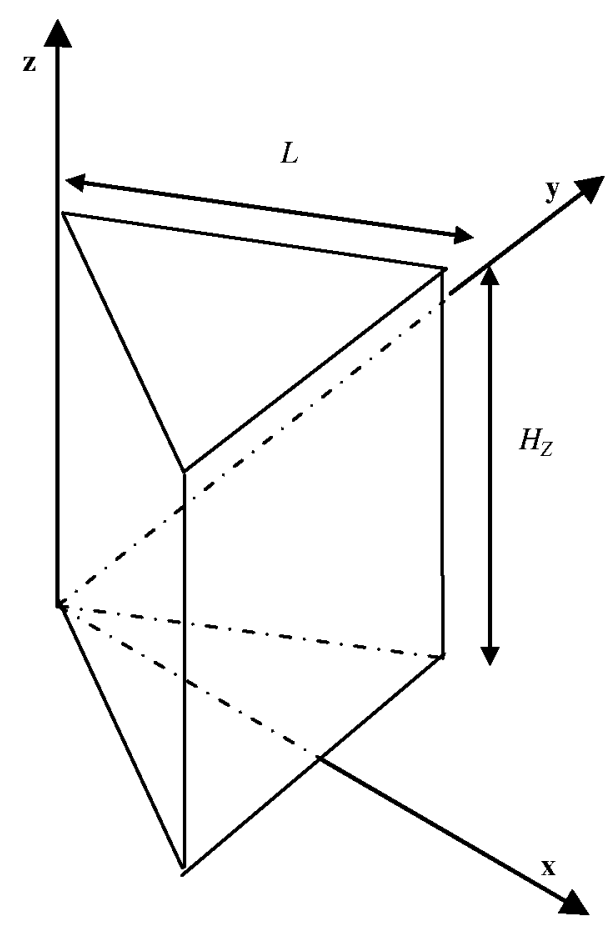

Fig. 1. Node in triangular-Z geometry (height $H_{z}$, triangle side length $L$ ). 
Let us consider the transverse integration over the $x$ direction. Integrating Eq. (1) over a slice of width $d x$ perpendicular to the $x$ axis.

$$
\begin{aligned}
& \int_{A(x)} j_{m}^{x} \cdot d y d z-\int_{A(x+d x)} j_{m}^{x} \cdot d y d z-\int_{P(x)} j_{m}^{n} \cdot d s-\lambda_{m} \int_{V} \psi_{m}(\vec{r}) \cdot d V \\
& \quad=-\int_{V} s_{m}(\vec{r}) \cdot d V
\end{aligned}
$$

where $j_{m}^{x}(\vec{r})=\frac{\partial \psi_{m}(\vec{r})}{\partial x}$ and $j_{m}^{n}(\vec{r})$ is the component of the modal current perpendicular to the peripheral area $P(x)$. Then it is easy to see that

$$
\begin{gathered}
{\left[\frac{d\left(\int_{A(x)} \frac{\partial \psi_{m}}{\partial x} \cdot d y d z\right)}{d x}-\frac{2}{\sqrt{3}} \int_{0}^{H_{z}}\left(j_{m}^{n+}+j_{m}^{n-}\right) \cdot d z-\int_{-a x}^{a x}\left(j_{m}^{z+}+j_{m}^{z-}\right) \cdot d y\right.} \\
\left.-\lambda_{m} \int_{A(x)} \psi_{m}(\vec{r}) \cdot d y d z\right] \cdot d x=\left[-\int_{A(x)} s_{m}(\vec{r}) \cdot d y d z\right] \cdot d x
\end{gathered}
$$

where $j_{m}^{n+}=j_{m}^{n}\left(x, \frac{x}{\sqrt{3}}, z\right), j_{m}^{n-}=j_{m}^{n}\left(x,-\frac{x}{\sqrt{3}}, z\right), j_{m}^{z+}=j_{m}^{z}\left(x, y, H_{z}\right)$ and $j_{m}^{z-}=j_{m}^{z}(x, y, 0)$.

Applying the Leibniz' rule to the first term of Eq. (3) we find that

$$
\begin{aligned}
\int_{A(x)} \frac{\partial \psi_{m}}{\partial x} \cdot d y d z= & \frac{d\left(\int_{A(x)} \psi_{m} \cdot d y d z\right)}{d x}-\frac{1}{\sqrt{3}}\left(\int _ { 0 } ^ { H _ { z } } \left(\psi_{m}\left(x, \frac{x}{\sqrt{3}}, z\right)\right.\right. \\
& \left.\left.+\psi_{m}\left(x,-\frac{x}{\sqrt{3}}, z\right)\right) \cdot d z\right)
\end{aligned}
$$

Substituting this expression into Eq. (3), we finally obtain the following equation over the flux integrated in the slice:

$$
\begin{aligned}
& \frac{d^{2} \psi_{m}^{1 D}(x)}{d x^{2}}-\lambda_{m} \psi_{m}^{1 D}(x)=-s_{m}^{1 D}(x)+L_{m}(x) \\
& \psi_{m}^{1 D}(x)=\int_{0}^{H_{Z}}\left[\int_{-a x}^{a x} \psi_{m}(x, y, z) \cdot d y\right] \cdot d z ; \quad a=\frac{1}{\sqrt{3}} \\
& s_{m}^{1 D}(x)=\int_{0}^{H_{Z}}\left[\int_{-a x}^{a x} s_{m}(x, y, z) \cdot d y\right] \cdot d z
\end{aligned}
$$

$L_{m}(x)$ is a term equivalent to the transverse leakage in Cartesian nodes, although in this case it has a major complexity:

$$
\begin{aligned}
L_{m}(x)= & \frac{2}{\sqrt{3}} \int_{0}^{H_{Z}}\left(j_{m}^{n+}+j_{m}^{n-}\right) \cdot d z-\frac{2}{3} \int_{0}^{H_{Z}}\left(j_{m}^{t+}+j_{m}^{t-}\right) \cdot d z \\
& +\int_{-a x}^{a x}\left(j_{m}^{z+}+j_{m}^{z-}\right) \cdot d y
\end{aligned}
$$

where $j_{m}^{t+}$ and $j_{m}^{t-}$ are the modal flux derivatives along the tangential direction to the radial interfaces (see Fig. 2).

As it can be seen, as occurs in Cartesian geometry nodes, the transverse-integrated flux is the unknown of the resulting $1 \mathrm{D}$ diffusion equation obtained after integration of the original 3D equation along a radial direction. It is important to clarify that, now, the

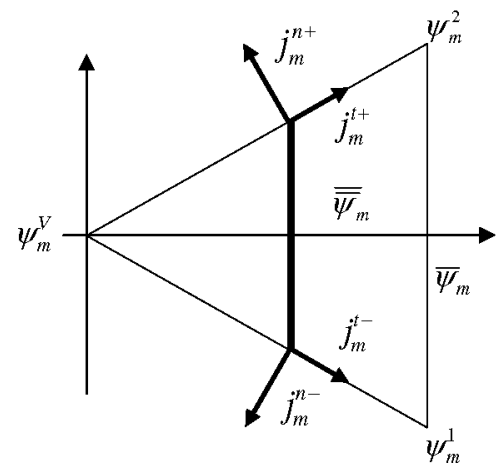

Fig. 2. Components of the modal current in the $X Y$ plane and magnitudes involved in the ACMFD relation. radial transverse leakage does not fit the net leakage through the side faces of the prism and a tangential component of current arises as a consequence of the non-constant transverse area. However, we will maintain its name as it plays the same role as in the case of Cartesian geometry.

Following, it will be shown how the ACMFD relation at a radial interface in triangular- $Z$ geometry can be derived, that is, how to relate both node surface currents and fluxes to node-average fluxes.

\subsection{The ACMFD relation at radial interfaces in $2 D$ triangular nodes}

For simplicity, we will consider first the 2D geometry case, that is, the triangular node shown in Fig. 2. As it can be seen in Eq. (5), the transverse-integrated flux being solution of a 1D diffusion equation can be expressed as a combination of a homogeneous solution (independent on the external source) and a particular solution $\left(p_{m}(x)\right)$ given by the function expansion of $L_{m}(x)$. This can be seen in the following equation:

$\psi_{m}^{1 \mathrm{D}}(x)=A_{m} \mathrm{e}^{+\alpha_{m} x}+B_{m} \mathrm{e}^{-\alpha_{m} x}+p_{m}(x) ;$

$\frac{d^{2}}{d x^{2}} p_{m}(x)-\lambda_{m} p_{m}(x)=L_{m}(x) ; \quad \alpha_{m}=\sqrt{\lambda_{m}}$

Particularizing expression (8) in the interface at the right side of the triangle (see Fig. 2 ) we realize that average flux at this interface can be related to constants $A_{m}$ and $B_{m}$ as follows:

$L \cdot \bar{\psi}_{m}\left(\frac{\sqrt{3}}{2} L\right)=A_{m} e^{\gamma}+B_{m} e^{-\gamma}+p_{m}\left(\frac{\sqrt{3}}{2} L\right) ; \quad \gamma=\frac{\sqrt{3}}{2} L \alpha_{m}$

In addition it is possible to particularize the first derivative of expression (8) at the same position. The objective of this operation is to get a relation between the interface average current and the constants $A_{m}$ and $B_{m}$. At this point, it is important to give more details about this derivation. On the one side it is possible to write:

$\frac{d \psi_{m}^{1 D}\left(\frac{\sqrt{3}}{2} L\right)}{d x}=\alpha_{m}\left(A_{m} e^{\gamma}-B-m e^{-\gamma}\right)+p_{m}^{\prime}\left(\frac{\sqrt{3}}{2} L\right)$

On the other side we have to take into account from (6) that $\psi_{m}^{1 D}(x)$ is an integral function with an interval (transverse area) depending on $x$ coordinate. For this we will have to apply Leibniz' rule:

$$
\begin{aligned}
\frac{d\left(\int_{y}^{y \frac{x}{\sqrt{3}}} \frac{x}{\sqrt{3}} \psi_{m}(x, y) \cdot d x\right)}{d x}= & \int_{y-\frac{x}{\sqrt{3}}}^{y-\frac{x}{\sqrt{3}}} \frac{\partial \psi_{m}(x, y)}{d x} \cdot d x \frac{1}{\sqrt{3}} \psi_{m}\left(x, \frac{1}{\sqrt{3}}\right) \\
& +\frac{1}{\sqrt{3}} \psi_{m}\left(x, \frac{1}{\sqrt{3}}\right)
\end{aligned}
$$

Simplifying the notation, the following expression is obtained:

$\frac{d \psi_{m}^{1 D}\left(\frac{\sqrt{3}}{2} L\right)}{d x}=\frac{1}{\sqrt{3}}\left(\psi_{m}^{1}+\psi_{m}^{2}\right)-L \bar{j}_{m}\left(\frac{\sqrt{3}}{2} L\right)$

where $\bar{j}_{m}$ is the modal average current at the interface and $\psi_{m}^{1}$ and $\psi_{m}^{2}$ are the modal fluxes at the corners adjacent to the interface.

Equating expressions (10) and (12) and taking into account also expression (9), constants $A_{m}$ and $B_{m}$ are constants that can be determined by imposing the following conditions: the modal interface average flux at a radial interface $\bar{\psi}_{m}$, the modal average current at the same interface $\bar{j}_{m}$ and the modal fluxes at the corners adjacent to the interface $\psi_{m}^{1}$ and $\psi_{m}^{2}$. 


$$
\begin{aligned}
A_{m}= & \frac{L}{2 e^{\gamma}} \bar{\psi}_{m}+\frac{1}{2 \sqrt{3} \alpha_{m} e^{\gamma}}\left(\psi_{m}^{1}+\psi_{m}^{2}\right)-\frac{L}{2 \alpha_{m} e^{\gamma}} \bar{j}_{m} \\
& -\frac{p_{m}\left(\frac{\sqrt{3}}{2} L\right)}{2 e^{\gamma}}-\frac{p_{m}^{\prime}\left(\frac{\sqrt{3}}{2} L\right)}{2 \alpha_{m} e^{\gamma}} \\
B_{m}= & \frac{L}{2 e^{-\gamma}} \bar{\psi}_{m}-\frac{1}{2 \sqrt{3} \alpha_{m} e^{-\gamma}}\left(\psi_{m}^{1}+\psi_{m}^{2}\right)+\frac{L}{2 \alpha_{m} e^{-\gamma}} \bar{j}_{m} \\
& -\frac{p_{m}\left(\frac{\sqrt{3}}{2} L\right)}{2 e^{-\gamma}}+\frac{p_{m}^{\prime}\left(\frac{\sqrt{3}}{2} L\right)}{2 \alpha_{m} e^{-\gamma}}
\end{aligned}
$$

Then, substituting in the node-average modal flux $\overline{\bar{\psi}}_{m}$, the ACMFD modal relation at every radial interface in triangular mesh is obtained:

$$
\begin{aligned}
\left(\bar{\psi}_{m}-\frac{p_{m}\left(\frac{\sqrt{3}}{2} L\right)}{L}\right)= & \frac{C_{m}^{f}}{2}\left(\overline{\bar{\psi}}_{m}-\frac{2 \bar{p}_{m}}{L}\right)+\frac{C_{m}^{j}}{4}\left(\psi_{m}^{1}+\psi_{m}^{2}\right) \\
& -\frac{\sqrt{3}}{4} L C_{m}^{j}\left(\bar{j}_{m}+\frac{p_{m}^{\prime}\left(\frac{\sqrt{3}}{2} L\right)}{L}\right)
\end{aligned}
$$

where $L$ is the triangle side length and $C_{m}^{f}$ and $C_{m}^{j}$ are the analytic coefficients defined as:

$C_{m}^{f}=\frac{2 \gamma}{e^{\gamma}-e^{-\gamma}} ; \quad C_{m}^{j}=\frac{e^{\gamma}+e^{-\gamma}-2}{e^{\gamma}-e^{-\gamma}} \cdot \frac{2}{\gamma}$

With the purpose of deriving a "half node" coupling equation, we only need to compute the average value of the particular solution $\left(\bar{p}_{m}\right)$ and the value of the function $\left(p_{m}\left(\frac{\sqrt{3}}{2} L\right)\right)$ and its derivative $\left(p_{m}^{\prime}\left(\frac{\sqrt{3}}{2} L\right)\right)$ in the right extreme. All these values will be given by the approach taken for the transverse leakage profile.

Let us compare expression (14) with the equivalent relation in Cartesian geometry, for the right side in a square node of width $H$ :

$\left(\bar{\psi}_{m}-p_{m}(H)\right)=C_{m}^{f}\left(\overline{\bar{\psi}}_{m}-\bar{p}_{m}\right)-\frac{H}{2} C_{m}^{j}\left(\bar{j}_{m}+p_{m}^{\prime}(H)\right)$

First, it is remarkable the difference in the terms containing the particular solution. In Cartesian geometry they are not divided by the triangle side as they come from a transverse-integrated equation already divided by the constant transverse area, so that the transverse leakage and the particular solution implicitly are also divided by $H$ (transverse area in the $2 \mathrm{D}$ case).

We can see that the main drawback in the triangular case is the presence of the modal fluxes at the two corners adjacent to the interface, which have to be computed with a level of accuracy appropriate to this high order method.

\subsection{The analytic relation at corners in $2 D$ triangular nodes}

To obtain the modal flux at a corner, we proceed as follows. We can particularize expression (8) at the coordinate $(x=0)$, which corresponds to the triangle vertex opposed to the analysed interface.

$\psi_{m}^{1 D}(0)=0=A_{m}+B_{m}+p_{m}(0)$

$\frac{d \psi_{m}^{1 D}}{d x}(0)=\frac{2}{\sqrt{3}} \psi_{m}^{V}=\alpha_{m}\left(A_{m}-B_{m}\right)+p_{m}^{\prime}(0)$

$\frac{\sqrt{3}}{4} L^{2} \overline{\bar{\psi}}_{m}=\frac{A_{m}}{\alpha_{m}}\left(e^{\gamma}-1\right)-\frac{B_{m}}{\alpha_{m}}\left(e^{-\gamma}-1\right)+\frac{\sqrt{3}}{4} L \bar{p}_{m}$

If we focus on the values of the function (17) and its first derivative (18) at this coordinate and substitute them in the expression for the nodal average flux (19), then we will obtain the following relation between the flux at vertex and the nodal average flux results:
$\left(\psi_{m}^{V}-\frac{\sqrt{3} \cdot p_{m}^{\prime}(0)}{2}\right)=B_{m}^{f}\left(\overline{\bar{\psi}}_{m}-\frac{2 \cdot \bar{p}_{m}}{L}\right)+B_{m}^{i} \frac{p_{m}(0)}{L}$

where $B_{m}^{f}$ and $B_{m}^{i}$ are analytic constants defined by the square root of the modal eigenvalue $\lambda_{m}$ and by the length of the triangle side $(L)$.

$B_{m}^{f}=\frac{\gamma^{2}}{e^{\gamma}+e^{-\gamma}-2} ; \quad B_{m}^{j}=\frac{\gamma \cdot\left(e^{\gamma}-e^{-\gamma}\right)}{e^{\gamma}+e^{-\gamma}-2} ; \quad \gamma=\frac{\sqrt{3}}{2} \alpha_{m} L$

2.4. Combination of the ACMFD relation at a radial interface with the analytic relations at the adjacent comers

Until now, it has been demonstrated that, as occurs in Cartesian geometry nodes, it also exists an ACMFD relation for triangular nodes (14). Nevertheless, we have also found that the flux particularized at adjacent vertexes takes part in such ACMFD relation.

The most straightforward option to treat Eq. (14) would be to take the term of fluxes at vertex from the previous iteration, including them in the non-linear iteration loop (as done with the transverse leakage term). However, this option is unfeasible as the iterative process becomes unstable because of the excessive influence of the transverse leakage term. Other possibility would be to set, in addition to the nodal average fluxes, the vertex fluxes as unknowns in the linear system, by introducing the corner-point balance equations. Nevertheless, in order to preserve the efficiency of the ACMFD method in Cartesian geometry, where the linear system includes the nodal average fluxes as the unique unknowns, the additional relation (20) is applied to eliminate the corner-point fluxes from the final linear system.

Consequently, it is necessary to substitute the flux at vertexes in relation (14) by their corresponding relation with the nodal average flux (20). Computing and grouping together the transverse leakage terms, we obtain the following expression:

$\bar{\psi}_{m}=C_{m}^{f} \overline{\bar{\psi}}_{m}-\frac{\sqrt{3}}{4} L C_{m}^{j} \bar{j}_{m}-T_{m}$

where the $T_{m}$ includes all terms related to the particular solution driven by the transverse leakages.

This way we finally have an expression quite similar to the ACMFD for Cartesian nodes, that is, the nodal equations are essentially the same in triangular and Cartesian geometries. In this case, the transverse leakage profile has a more complex physical meaning than in the previous one.

However, this approach (in which nodal average fluxes are the unique unknowns) will not lead to the level of accuracy achieved with Cartesian geometry. The reason of this assertion is that the accuracy of the analytic nodal method relies on a good estimation of the average value of the transverse leakage. In Cartesian mesh this average value only depends on the interface average currents whose errors vary from $0.1 \%$ to $1 \%$ in most of nodal solutions. Nevertheless attending to the transverse leakage expression in triangular mesh (7), we can see that transverse leakage not only gathers the perpendicular component of current to the surface, but also the tangential one. This will force us to compute the flux at the three vertexes of every node.

Basically the fluxes at corners are computed using the cornerpoint balance equations, involving the intranodal flux distributions of the six triangular nodes adjacent to the considered vertex. With this aim we use the analytic expression (20) for each one of the six nodes, obtaining a relation between flux at a given corner and the six nodal average fluxes. Thus the corner fluxes are iteratively updated in transverse leakage loop, once the nodal average fluxes and the interface average fluxes and currents have been computed.

For both analytic relations at interfaces (14) and vertex (20) a polynomial profile has been assumed for the transverse leakage 
$L_{m}(x)$. Particularly a cubic fit has been adopted, which has four coefficients that are determined guessing the values of $\bar{L}_{m}, L_{m}(0), L_{m}\left(\frac{\sqrt{3}}{2} L\right)$ and one of the values of $L_{m}^{\prime}(0), L_{m}^{\prime}\left(\frac{\sqrt{3}}{2} L\right)$. To compute these values a parabolic interpolation of the average currents at radial interfaces has been adopted.

\subsection{Extension of $A C M F D$ theory to $3 D$ (triangular- $Z$ geometry)}

In triangular- $Z$ geometry, the main difficulties arise from the fact that the transverse area changes in the radial direction. Once provided the formulation for $2 \mathrm{D}$ triangular nodes, the extension to the 3D case is not difficult. We will have to overcome two additional aspects. First, an ACMFD relation for axial top and bottom interfaces has to be derived. Second, the transverse leakage has to be redefined to include the axial component as well, that is, the neutron leakage through the top and bottom interfaces has to be taken into account in the particular solution of Eq. (14), then the transverse leakage $L_{m}(x)$ is modified.

\subsubsection{The ACMFD relation for axial interfaces}

As it has been explained in previous sections, the first step to obtain an ACMFD relation is to perform a transverse integration of the 3D diffusion equation. In this case the integration is over the triangular area perpendicular to the $z$ axis. In axial direction, the transverse area is constant, so the procedure to obtain the ACMFD relation is the same as in Cartesian nodes. Starting from the $3 \mathrm{D}$ modal equations we obtain the $1 \mathrm{D}$ modal equation over the $1 \mathrm{D}$ averaged flux:

$$
\begin{aligned}
& \frac{d^{2} \psi_{m}^{1 D}(z)}{d z^{2}}-\lambda_{m} \psi_{m}^{1 D}(z)=-s_{m}^{1 D}(z)+L_{m}(z) \\
& L_{m}(z)=\frac{4}{\sqrt{3} L} \sum_{r=1}^{3} j_{m, r}(z)
\end{aligned}
$$

where $j_{m, r}(z)$ is the averaged outgoing current through radial interfaces at height $z$.

Independently of the way we obtain the transverse leakage $L_{m}$ (z), Eq. (23) leads to an ACMFD relation similar to the Cartesian one with the only particularity that the transverse leakage is computed in a different way.

2.5.2. Modifications of the ACMFD relation for radial interfaces in $3 D$ nodes

In 3D nodes, Eq. (7) can be written in the following way:

$$
\begin{aligned}
L_{m}(x) & =L_{m}^{R}(x)+\int_{-a x}^{a x}\left(j_{m, z}\left(x, y, H_{z}\right)-j_{m, z}(x, y, 0)\right) \cdot d y \\
& =L_{m}^{R}(x)+L_{m}^{Z}(x)
\end{aligned}
$$

where $L^{R}$ and $L^{Z}$ refers to leakage through radial and axial interfaces, respectively.

The transverse leakage profile in $z$-direction leads to an additional particular solution $\left(p^{Z}\right)$. Then Eq. (8) is modified:

$\psi_{m}^{1 D}(x)=A_{m} \mathrm{e}^{+\alpha_{m} x}+B_{m} \mathrm{e}^{-\alpha_{m} x}+p_{m}^{R}(x)+p_{m}^{Z}(x)$

$\frac{d^{2}}{d x^{2}} p_{m}^{Z}(x)-\lambda_{m} p_{m}^{Z}(x)=L_{m}^{Z}(x)$

Finally it is worthy to remark that if we want to assume a polynomial profile for $L_{m}^{Z}(x)$, we will have to take into account that the integration length in (24) varies with $x$.

\subsection{The ACMFD method for kinetics problems}

Under transient conditions, both the time-dependent multigroup diffusion Eq. (26) and the balance equations of the six neutron precursors (27) have to be solved together. The idea is to treat the temporal dependence in such a way that it yields a fixed source problem (FSP), which can be solved utilizing the methodology already developed for the steady-state FSP. This methodology is independent on the geometry so it can be applied to triangular- $Z$ nodes with slight modifications regarding Cartesian nodes (Lozano et al., 2008b).

The neutron-kinetics equations can be written in a matricial way as follows:

$\left|\frac{1}{D_{g} \mathbf{v}_{g}} \frac{\partial \phi_{g}}{\partial t}\right\rangle=\nabla^{2}\left|\phi_{g}\right\rangle-\mathbf{A}\left|\phi_{g}\right\rangle-\mathbf{F}_{\mathbf{d}}\left|\phi_{g}\right\rangle+\sum_{k=1}^{6} \lambda_{k} C_{k}\left|\frac{\chi_{d_{k g}}}{D_{g}}\right\rangle$

$\frac{\partial C_{k}}{\partial t}=f_{k} \beta \sum_{g=1}^{G} \frac{v \sum_{f}^{g}}{k_{e f f}} \phi_{g}-\lambda_{k} C_{k} \quad$ for $k=1, \ldots, 6$

where $\boldsymbol{A}$ is the steady-state multigroup diffusion matrix, $\chi$ and $\chi_{d}$ refer to the prompt and delayed neutron spectrum, respectively, and the $i g-j g$ element of the $\boldsymbol{F}_{\boldsymbol{d}}$ matrix is defined as $F_{d}(i g, j g)=\frac{\beta \beta^{i g}}{D_{i g}} \frac{v \Sigma_{f}^{i g}}{k_{e f f}}$. The rest of terms are in standard notation.

To treat the time dependence, the first step is to discretize the time domain into discrete time steps. Focusing on the neutron precursor balance equation and using a finite difference implicit scheme for the time derivative (neutron precursor concentrations are assumed to have smooth variations with time steps employed in-core transient analysis), we can obtain a relation between every precursor concentration and the prompt neutron flux per energy group:

$C_{k}(t, \vec{r})=\frac{C_{k}(t-\Delta t, \vec{r})+\Delta t \cdot f_{k} \beta \sum_{g=1}^{G} v \Sigma_{f g} \phi_{g}(t, \vec{r})}{1+\lambda_{k} \Delta t}$

On the other hand, to approximate the time derivative of the neutron-kinetics equation, we use the method of exponential extrapolation, $\phi_{g}(r, t)=\tilde{\phi}_{g}(r, t) \cdot e^{\omega_{g} t}$, and then the time derivative of the form function $\tilde{\phi}_{g}$ is approximated by an implicit linear forward difference scheme, which is appropriate for most of the nodes (those that are far from moving control rods). Thus the neutron flux time derivative can be written as follows:

$$
\begin{aligned}
\frac{1}{D_{g} \mathrm{v}_{g}} \frac{\partial \phi_{g}(t, \vec{r})}{\partial t}= & \left(\frac{\omega_{g}}{D_{g} \mathrm{v}_{g}}+\frac{1}{D_{g} \mathrm{v}_{g} \Delta t}\right) \cdot \phi_{g}(t, \vec{r}) \\
& -\frac{1}{D_{g} \mathrm{v}_{g} \Delta t} \cdot \phi_{g}(t-\Delta t, \vec{r}) \cdot e^{\omega_{g} \Delta t}
\end{aligned}
$$

The frequency $\omega_{g}$ is recursively computed for each node and energy group using the nodal average flux:

$\omega_{g}=\frac{1}{\Delta t} \ln \left(\frac{\bar{\phi}_{g}(t)}{\bar{\phi}_{g}(t-\Delta t)}\right)$

The iterative process over the frequency $\omega_{g}$ is performed until the required convergence in the fission source distribution is achieved.

The use of the exponential extrapolation method strongly improves the efficiency of the solver in transient calculations where the flux evolution follows the fundamental mode. This occurs in all those phases of the transients when there are no relevant changes in neutronic properties (control rod movement, thermal-hydraulic conditions, etc.). Hence, this approach allows to increase the transient time steps in these phases.

Regarding the spectrum of delayed neutrons, it is known that it is softer than the prompt fission spectrum. However, henceforward it will be assumed the same neutron spectrum for prompt $(\chi)$ and delayed neutrons $\left(\chi_{d}\right)$, as specified in the benchmark presented in this paper.

Introducing those approximations into the balance equations of neutron precursors and into Eq. (26): 
$\nabla^{2}\left|\phi_{g}\right\rangle-\mathbf{A}\left|\phi_{g}\right\rangle-\mathbf{A}_{\mathbf{k i n}}\left|\phi_{g}\right\rangle=-\left|\frac{e^{\omega_{g} \Delta t}}{D_{g} \mathrm{~V}_{g} \Delta t} \phi_{g}^{0}\right\rangle-\left(\sum_{k=1}^{6} \frac{\lambda_{k} C_{k}^{0}}{1+\lambda_{k} \Delta t}\right)\left|\frac{\chi^{g}}{D_{g}}\right\rangle$

$A_{\text {kin }}($ ig,$j g)=\frac{1}{D_{i g}}\left[\left(\sum_{k=1}^{6} \frac{\Delta t f_{k}}{1+\lambda_{k} \Delta t}\right) \chi^{i g} \beta v \Sigma_{f}^{j g}+\delta_{i g, j g}\left(\frac{\omega_{i g}}{v_{i g}}+\frac{1}{v_{i g} \Delta t}\right)\right]$

We can see that Eq. (31) is a FSP having additional terms with respect to the steady-state FSP: the multigroup matrix is now $\boldsymbol{A}+\boldsymbol{A}_{\boldsymbol{k i n}}$, and the external source has an additional term determined by both the flux spatial distribution and the precursor concentrations in the previous time step $\left(\phi_{g}^{0}, C_{k}^{0}\right)$.

In order to obtain an accurate ACMFD relation for transient problems, the most important is to decide which terms are included in the multigroup diffusion matrix $\left(\boldsymbol{A}+\boldsymbol{A}_{\boldsymbol{k i n}}\right)$ and the spatial distribution assigned to the terms included in the external source.

\section{The NK-TH coupling scheme for hexagonal problems}

The neutron-kinetics (NK) and TH coupling in ANDES/COBRA-IIIC was originally developed for 3D Cartesian geometry (Jiménez et al., 2007,2008 ), including the capability of refining the radial mesh by using both one or four neutronic nodes and one or four channels per fuel assembly. The use of the TH channel code COBRA-IIIC in LWR, provides an accurate estimation of the in-core 3D flow distribution due to the modelling of the diversion cross flow and turbulent mixing between adjacent channels, in the solution of the momentum equation in the radial direction.

The next step in the coupling scheme development has been its extension to VVER cores by addressing the challenging hexagonal mesh geometry of this type of reactors. The objective is to maintain the capability of performing a radial mesh refinement (see Fig. 3), and to take advantage of the cross flow treatment to provide an accurate estimation of the 3D flows also in hexagonal geometry problem applications.

With this aim, the NK-TH coupling extension to hexagonal geometry has been recently carried out. The coupled ANDES/CO$B R A$-IIIC code has the capability to model the core using either assembly-wise channels (hexagonal channels) or six channels (triangular channels) per fuel assembly if a higher level of refinement is required in the TH radial nodalization. Regarding the axial meshing, the only restriction to the node height is related to the stability of both NK and TH solvers. The required stability imposes a number of axial nodes varying between a minimum value of ten axial layers up to forty, depending on the strength of the feedback in the axial direction. In the TH core modelling of VVER-1000 Coolant Transient Benchmark (Ivanov et al., 2004), most of participants considered just twelve axial layers and several fuel assemblies were represented by a single homogenized $1 \mathrm{D}$ channel. The capability of the TH mesh refinement with COBRA-IIIC code allows to improve the TH VVER core modelling, providing a better estimation of the in-core 3D flow distribution. This will be demonstrated in Section 4.

Regarding the NK-TH time coupling, it is carried out using a staggered alternate time mesh scheme (Merino et al., 1993). The most remarkable fact of the algorithm employed is that the calculations of $\mathrm{TH}$ and NK variables do not coincide in time. By means of this scheme, the energy is conserved in first order. For linear variations of power, the energy is exactly conserved, while for sharp exponential variations, a factor must be included to correct the linear approximation.

The time coupling scheme has been successfully implemented and shows an accurate and robust performance, with minimal diffusion and oscillations. The time step size is supplied by the user through input deck. It allows the detailed analysis of the different transient stages by selecting small time step sizes as well as the acceleration of the calculation by using large time steps when the changes in $\mathrm{NK}$ and $\mathrm{TH}$ variables are not so relevant. A numerical analysis has shown that the stability limit of this coupling scheme is reached when a time step bigger than approx. 0.8$1.0 \mathrm{~s}$ is imposed for coupled calculations at nominal conditions.

\section{Verification results}

The verification of the ANDES/COBRA-IIIC extension to hexagonal geometry and its adequacy to the analysis of coupled transient has been carried out using the V1000CT2-EXT2 (Kolev et al., 2007a) and the Exercise 2 of the V1000CT-1 Benchmark (Ivanov et al., 2004). In order to model properly the fuel behaviour in the above exercises, a polynomial fitting for the fuel, gap and cladding properties (thermal conductivity and heat capacity) was developed from the benchmark specifications to take into account their dependence on temperature. Those third order polynomials were implemented as correlations within the code COBRA-IIIC.

Table 1 presents the results corresponding to different HZP steady state situations proposed in the V1000CT2-EXT2 Benchmark (Kolev et al., 2007a). All the cases correspond to different control rod insertions. Two results are provided for each single case, one using six neutronic nodes per fuel assembly and another using 24 neutronic nodes per fuel assembly. A comparison is performed using the average of the results given by PARCS (Downar et al., 2004) and DYN3D (Grundmann and Hollstein, 1999) codes as reference solution.

From Table 1, it can be figured out that the agreement of our coarser solution (COBAYA 6N) with the reference is quite good, and also consistent with the refined solution (COBAYA 24N). Namely, the differences in $k_{\text {eff }}$ are lower than 30 pcm even in the cases " 3 " and " 4 " which are highly asymmetric due to a control
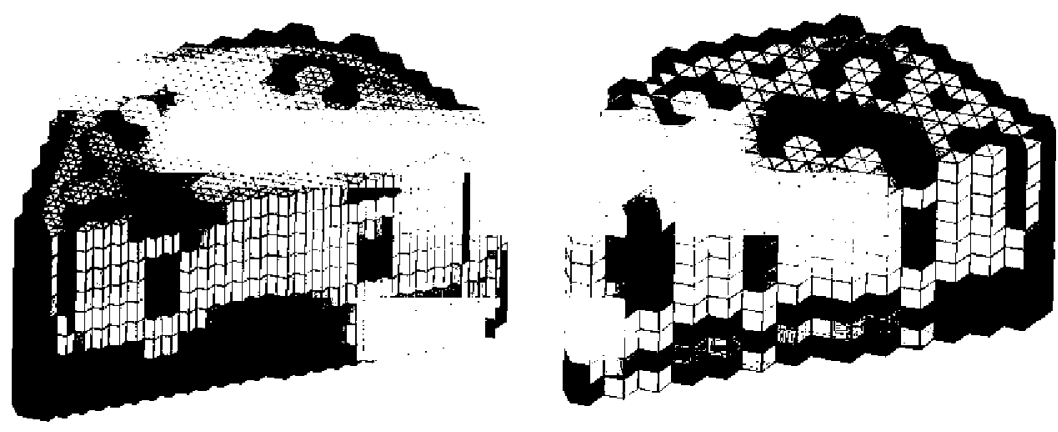

Fig. 3. Different mesh refinement capability applied to triangular-Z mesh. 
Table 1

Comparison of ANDES/COBRA-III results for the V1000CT2-EXT2 HZP cases versus PARCS and DYN3D.

\begin{tabular}{|c|c|c|c|c|c|}
\hline Case & & $K_{\text {eff }}$ & $F_{x y}$ & $F_{z}$ & A.O. (\%) \\
\hline \multirow[t]{3}{*}{0} & Reference & 1.02987 & 1.339 & 2.955 & 80.33 \\
\hline & COBAYA $6 \mathrm{~N}^{\mathrm{a}}$ & 1.03006 & 1.340 & 2.958 & 80.49 \\
\hline & COBAYA $24 \mathrm{~N}^{\mathrm{b}}$ & 1.03003 & 1.340 & 2.962 & 80.57 \\
\hline \multirow[t]{3}{*}{$1 \mathrm{a}$} & Reference & 0.99750 & 1.415 & 1.869 & 48.40 \\
\hline & COBAYA $6 \mathrm{~N}^{\mathrm{a}}$ & 0.99773 & 1.418 & 1.871 & 48.69 \\
\hline & COBAYA $24 \mathrm{~N}^{\mathrm{b}}$ & 0.99764 & 1.415 & 1.874 & 48.78 \\
\hline \multirow[t]{3}{*}{$1 b$} & Reference & 0.96203 & 1.385 & 2.340 & 63.70 \\
\hline & COBAYA $6 \mathrm{~N}^{\mathrm{a}}$ & 0.96223 & 1.388 & 2.325 & 63.62 \\
\hline & COBAYA $24 \mathrm{~N}^{\mathrm{b}}$ & 0.96216 & 1.383 & 2.334 & 63.91 \\
\hline \multirow[t]{3}{*}{3} & Reference & 0.96855 & 6.478 & 2.117 & 56.93 \\
\hline & COBAYA $6 \mathrm{~N}^{\mathrm{a}}$ & 0.96884 & 6.544 & 2.099 & 56.36 \\
\hline & COBAYA $24 \mathrm{~N}^{\mathrm{b}}$ & 0.96869 & 6.490 & 2.108 & 56.65 \\
\hline \multirow[t]{3}{*}{4} & Reference & 0.96854 & 6.485 & 2.112 & 56.75 \\
\hline & COBAYA $6 \mathrm{~N}^{\mathrm{a}}$ & 0.96883 & 6.551 & 2.094 & 56.20 \\
\hline & COBAYA $24 \mathrm{~N}^{\mathrm{b}}$ & 0.96868 & 6.495 & 2.103 & 56.49 \\
\hline
\end{tabular}

a $6 \mathrm{~N}: 6$ radial neutronic nodes per assembly.

b $24 \mathrm{~N}$ : 24 radial neutronic nodes per assembly.

rod stuck out of the core close to the periphery. The error in the radial peaking factor $\left(F_{x y}\right)$ is lower than $1 \%$ which is reduced to $0.2 \%$ with 24 nodes/FA. This demonstrates the high order of our ACMFD method, since it is possible to obtain very accurate results using a coarse mesh for hexagonal geometry (just six triangular nodes per fuel assembly).

The coupled code ANDES/COBRA-IIIC was also applied to solve the Scenario 2 (with return to power) of the V1000CT2-EXT2 Benchmark transient. This transient is initiated by a large MSLB at hot full power (HFP) which causes a deep and asymmetric core overcooling which is modelled by changing the TH boundary conditions at the core inlet. It is followed by a scram that starts at $0.36 \mathrm{~s}$ of the transient. The time for full insertion of the control rods is $4 \mathrm{~s}$. Following the scram signal, two of the most reactive peripheral control assemblies remain stuck out of the core, close to the location of maximum overcooling.

Figs. 4 and 5 show the ANDES/COBRA-IIIC results for this transient. They were obtained using six triangular nodes per assembly, 1 hexagonal channel for the TH and 30 axial layers for the active core.

ANDES/COBRA-IIIC solution is compared with the results of the OECD V1000CT-2 Benchmark participants (HEXTRAN/SMABRE, DYN3D/ATHLET, RELAP 3D) (Kolev et al., 2006, 2007b; Spasov et al., 2009).

Note that HEXTRAN/SMABRE, DYN3D/ATHLET and RELAP 3D results in Figs. 4 and 5 are from Exercise 2 of the OECD V1000CT-2 MSLB Benchmark, which is a vessel boundary conditions problem, and the V1000CT2-EXT2 Benchmark is a core boundary conditions

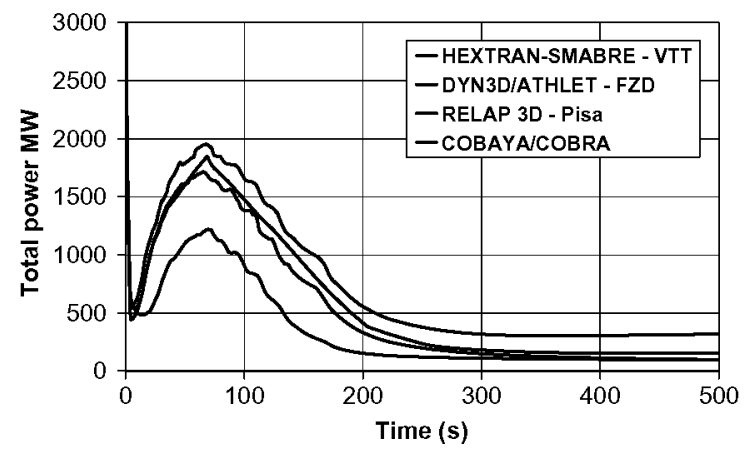

Fig. 4. Time history of total power in Scenario 2 of the V1000CT-2 Benchmark.

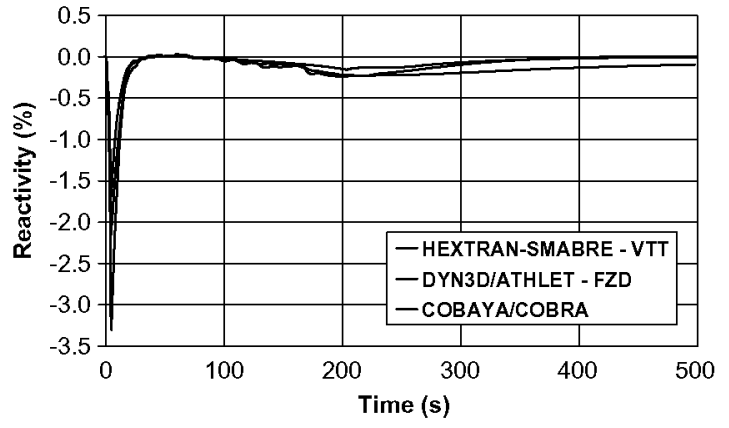

Fig. 5. Time history of reactivity in Scenario 2 of the V1000CT-2 Benchmark.

problem derived from V1000CT-2. The specifications of the two test problems are consistent but the core inlet $\mathrm{TH}$ boundary conditions in V1000CT2-EXT2 are pre-calculated and specified, while in V1000CT-2 they are calculated by the corresponding system code, given the vessel boundary conditions. Besides this difference, the time history of the total power matches up with the other solutions during the fall of the power and also in the return to power.

Next are presented the results obtained for the second exercise of the V1000CT-1 Benchmark (Ivanov and Ivanov, 2007). The steady states at Hot Zero Power (HZP) and Hot Power (HP) are summarized in Table 2 and Table 3, respectively. From here on, we will consider the average result of the benchmark participants, with its associated standard deviation, as the reference solution for our comparisons.

For the HZP steady state, our solution was obtained using ten axial layers within the active core plus two layers for the lower and upper axial reflectors. Furthermore six triangular nodes per fuel assembly were used for the neutronic radial nodalization. The results obtained for the HZP conditions show a very good agreement with the benchmark reference solution (they are within the standard deviation for all the parameters studied). Consequently, the extension of the nodal diffusion code ANDES to hexagonal geometry is demonstrated to be successful.

Regarding the HP steady state, four different Results (A, B, C, and $D$ ) are presented in Table 3, changing the mesh refinement in both neutronic and thermal-hydraulic sides. Result "A" was computed with the coarse mesh, using ten axial nodes, six triangular neutronic nodes and one hexagonal channel per fuel assembly. The results " $B$ " and " $C$ " had the same number of axial layers, 20, and the same TH meshing, one hexagonal channel per fuel assembly, but they had different neutronic nodalizations. Result "B" was obtained using six triangular nodes per fuel assembly while Result " $\mathrm{C}$ " used 24 triangular nodes per fuel assembly. Finally, Result " $D$ " was calculated with the finest meshing, using forty axial layers, with triangular channels (six channels per fuel assembly) and six triangular neutronic nodes per fuel assembly.

Looking at Table 3, most of the discrepancies are in the axial power distribution, which is due to the different axial nodalization of the participants. Most of them just used twelve axial layers, which explains why the Result "A" (with ten axial nodes) compares better against the reference solution in terms of Axial Offset (A.O.)

Table 2

ANDES/COBRA-III result for HZP steady state in Exercise 2.

\begin{tabular}{lllll}
\hline HZP case & $K_{\text {eff }}$ & $F_{x y}$ & $F_{z}$ & A.O. (\%) \\
\hline Reference result & 1.00022 & 1.416 & 1.519 & 16.88 \\
(Standard deviation) & $( \pm 0.00083)$ & $( \pm 0.012)$ & $( \pm 0.007)$ & $( \pm 0.84)$ \\
ANDES/COBRA-III & 0.99979 & 1.407 & 1.521 & 16.44 \\
(Deviation from the Ref.) & $(-0.00043)$ & $(-0.009)$ & $(+0.002)$ & $(-0.44)$ \\
\hline
\end{tabular}


Table 3

ANDES/COBRA-IIIc results for HP steady state with several nodalizations.

\begin{tabular}{|c|c|c|c|c|}
\hline HP steady state & $K_{\text {eff }}$ & $F_{x y}$ & $F_{z}$ & A.o. (\%) \\
\hline $\begin{array}{l}\text { REFERENCE RESULT } \\
\text { (Standard deviation) }\end{array}$ & $\begin{array}{l}0.99998 \\
( \pm 0.00116)\end{array}$ & $\begin{array}{l}1.341 \\
( \pm 0.011)\end{array}$ & $\begin{array}{l}1.406 \\
( \pm 0.021)\end{array}$ & $\begin{array}{l}16.29 \\
( \pm 1.28)\end{array}$ \\
\hline Result A $(6 \mathrm{~N} / 1 \mathrm{TH} / 10 \mathrm{Z})^{\mathrm{a}}$ & 1.00056 & 1.349 & 1.427 & 17.76 \\
\hline (Deviation from the Ref.) & $(+0.00058)$ & $(+0.008)$ & $(+0.021)$ & $(+1.47)$ \\
\hline $\begin{array}{l}\text { Result } \mathrm{B}(6 \mathrm{~N} / 1 \mathrm{TH} / 20 \mathrm{Z}) \\
\text { (Deviation from the Ref.) }\end{array}$ & $\begin{array}{l}1.00061 \\
(+0.00063)\end{array}$ & $\begin{array}{l}1.349 \\
(+0.008)\end{array}$ & $\begin{array}{l}1.431 \\
(+0.025)\end{array}$ & $\begin{array}{l}18.23 \\
(+1.94)\end{array}$ \\
\hline $\begin{array}{l}\text { Result C }(24 \mathrm{~N} / 1 \mathrm{TH} / 20 \mathrm{Z}) \\
\text { (Deviation from the Ref.) }\end{array}$ & $\begin{array}{l}1.00055 \\
(+0.00057)\end{array}$ & $\begin{array}{l}1.348 \\
(+0.007)\end{array}$ & $\begin{array}{l}1.431 \\
(+0.025)\end{array}$ & $\begin{array}{l}18.20 \\
(+1.91)\end{array}$ \\
\hline $\begin{array}{l}\text { Result } \mathrm{D}(6 \mathrm{~N} / 6 \mathrm{TH} / 40 \mathrm{Z}) \\
\text { (Deviation from the Ref.) }\end{array}$ & $\begin{array}{l}1.00053 \\
(+0.00055)\end{array}$ & $\begin{array}{l}1.347 \\
(+0.007)\end{array}$ & $\begin{array}{l}1.433 \\
(+0.027)\end{array}$ & $\begin{array}{l}18.44 \\
(+2.15)\end{array}$ \\
\hline
\end{tabular}

${ }^{a}(6 \mathrm{~N} / 1 \mathrm{TH} / 10 \mathrm{Z}): 6$ radial neutronic nodes, 1 thermal-hydraulics channel per fuel assembly, 10 axial layers.

and axial peaking factor $\left(F_{z}\right)$. Those results show the strong dependence of the axial profile on the TH feedback.

Regarding the radial power distribution, a comparison of that magnitude extracted from the Result " $B$ " (20 axial layers and one channel per fuel assembly) against the reference solution in one sector of the core is presented in Fig. 6. It can be seen that $A N$ $D E S / C O B R A-I I I C$ distribution is in good agreement with the reference solution under the HP steady state condition (our deviation is within the standard deviation for almost all the nodes). Therefore, it is proved that the extension to hexagonal geometry of the coupling scheme has been properly implemented.

After the steady state cases, we have run the guided transient using the boundary condition files provided with the benchmark documentation. In those files, the mass flow rate, coolant temperature and pressure conditions per fuel assembly versus time were specified. Because of the strong axial dependence of the TH feedback, in this exercise we used two different nodalizations: one using 20 axial layers and hexagonal channels, and other using forty axial layers and triangular channels. Both transients used the same neutronic nodalization radially (six triangles per fuel assembly). The computed results are presented in Figs. 7-9, where the mean values of the results obtained by the different participants in this transient exercise are also shown.

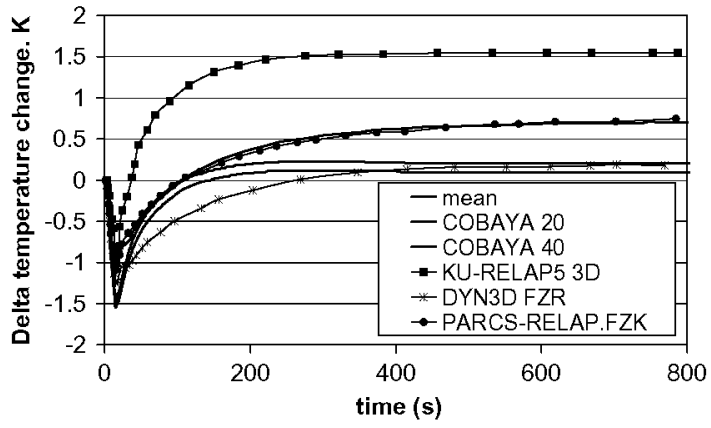

Fig. 7. Time history of core average fuel temperature.

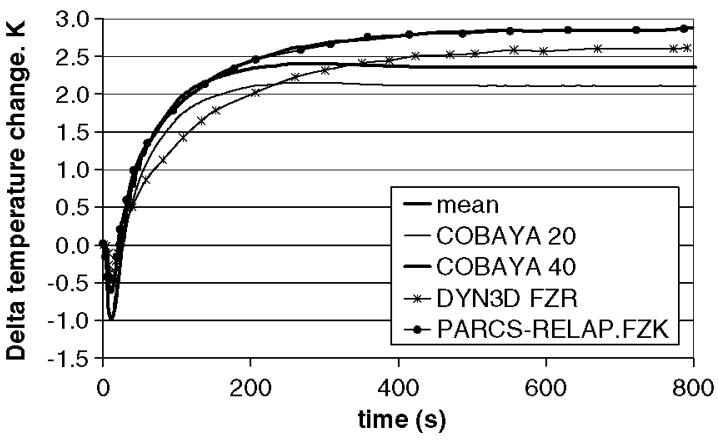

Fig. 8. Time history of maximum nodal average fuel temperature.

The deviations of the results with respect to the mean values could lead us to conclude that the performance in the transient calculation is not so good. To explain these discrepancies, we have included in the figures some representative results to show the wide spread of the solutions provided by the different participants. For instance, the delta power change at $800 \mathrm{~s}$ varies from 21 to $45 \mathrm{MW}$. Hence, it could be said that the mean value can not be taken as a reference solution and that the coupled code ANDES/CO$B R A-I I I C$ reproduces well the transient scenario.

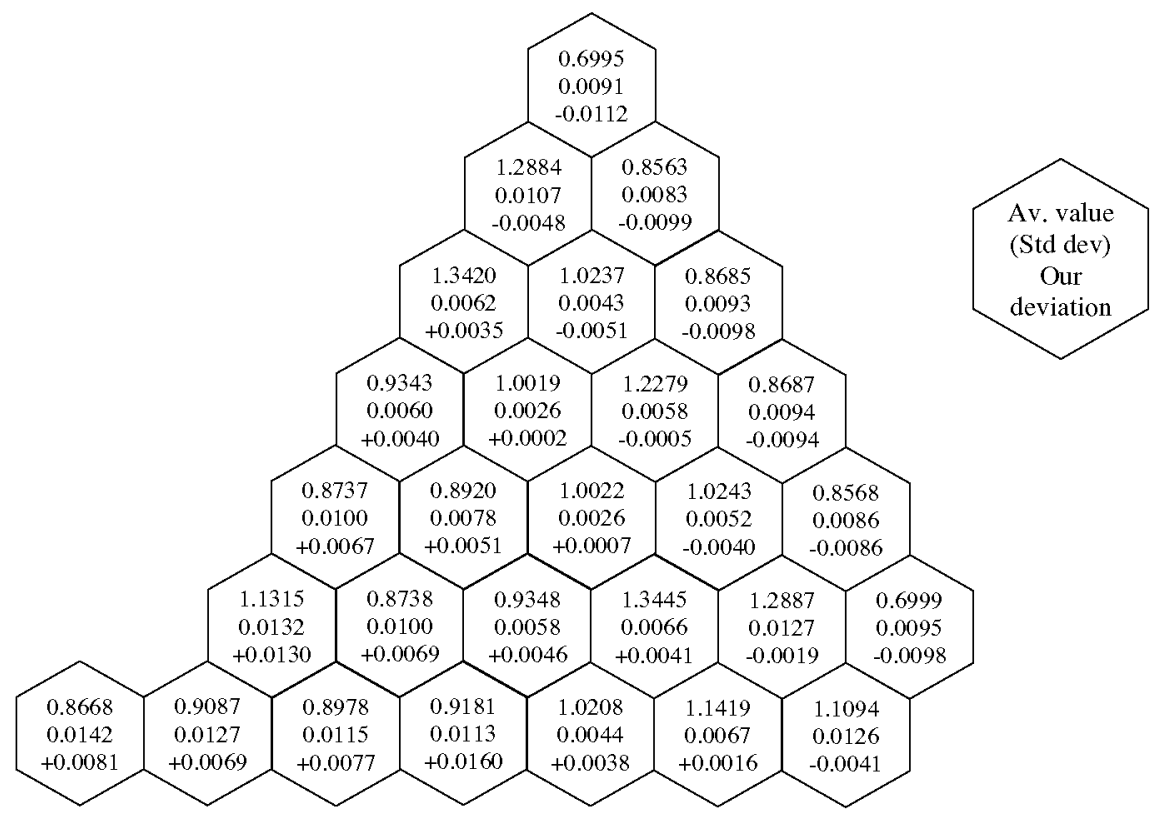

Fig. 6. Radial power distribution comparison for the HP steady state. 


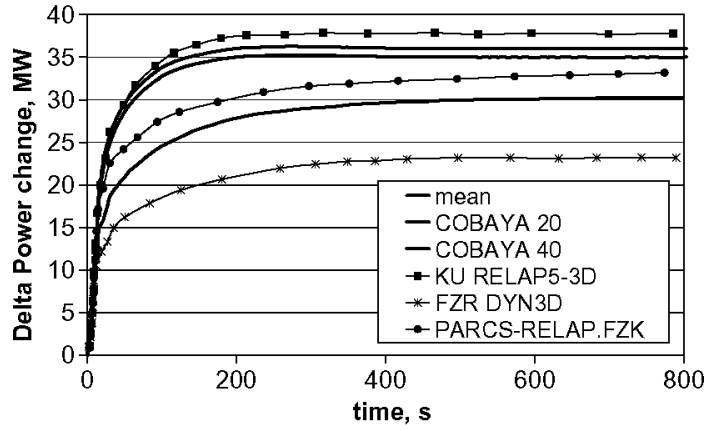

Fig. 9. Time history of power.

\section{Conclusions}

The coupled neutronics-thermal-hydraulics ANDES/COBRA-IIIC code has been extended to hexagonal geometry applications. In contrast to most of hexagonal nodal methods, it is based on direct transverse integration.

As for the neutronics, the extension of the ACMFD method has been carried out using triangular- $Z$ nodes, following the same guidelines of the method developed for Cartesian nodes. The used nodalization takes advantage of the mesh refinement capabilities implicitly when using this geometry instead of hexagonal nodes, and avoids the singularities that appear when applying transverse integration to hexagonal nodes. The results obtained for the HZP cases show the high order of our ACMFD method, since it is possible to obtain very accurate results using a coarse mesh for hexagonal geometry. The deviations in $k_{\text {eff }}$ are lower than $30 \mathrm{pcm}$ and the relative error in the assembly power is below $1 \%$.

As for the thermal-hydraulics, the coupling scheme has also been extended to hexagonal problems, with the capability to model the core using either hexagonal or triangular mesh. The use of COBRA-IIIC code with this level of TH mesh refinement provides an accurate estimation of the in-core 3D flow distribution.

The coupled code has been verified using two internationally well-known VVER benchmarks. The numerical results obtained for different steady-state and transient calculations have shown a quite good agreement with the reference solutions. We have confirmed that $A N D E S$ has a good reliability for the kinetic calculations of reactor cores with hexagonal fuel assemblies, and we have proved that the extension of the coupling scheme has been properly implemented.

This verification of the coupled code system can be considered as a promising starting point for the development of a simulation tool for the proposed Gen IV advanced core designs using hexagonal fuel assembly configurations.

\section{Acknowledgments}

This work is partially funded by the European Commission under the 6th EURATOM Framework Programme, within the RTD Integrated Projects NURESIM and NURISP, with contract numbers 516560 (FI60) and E080530-1042, respectively. The work of the first authors is part of their PhD thesis and has been supported by the Universidad Politécnica de Madrid and the Consejo de Seguridad Nuclear (Spain). We also thanks the collaboration of I. Spasov from IRNST during his stay at Universidad Politécnica de Madrid.

\section{Appendix A. Supplementary material}

Supplementary data associated with this article can be found, in the online version, at doi:10.1016/j.anucene.2009.12.001.

\section{References}

Aragonés, J.M., Ahnert, C., García-Herranz, N., 2007. The analytic coarse-mesh finitedifference method for multigroup and multidimensional diffusion calculations. Nucl. Sci. Eng. 157, 1-15.

Chao, Y.A., Shatilla, Y.A., 1995. Conformal mapping and hexagonal nodal methods Il: implementation in the ANC-H code. Nucl. Sci. Eng. 121, 210-225.

Chao, Y.A., Tsoulfanidis, N., 1995. Conformal mapping and hexagonal nodal methods - I: mathematical foundation. Nucl. Sci. Eng. 121, 202-209.

Chao, Y.A., 1999. A theoretical analysis of the coarse mesh finite difference representation in advanced nodal methods. In: Aragonés, J.M. (Ed.), Mathematics and Computation, Reactor Physics and Environmental Analysis in Nuclear Applications, vol. 1. Senda ed., Madrid, pp. 117-126.

Cho, N.Z., Noh, J.M., 1995. Analytic function expansion nodal method for hexagonal geometry. Nucl. Sci. Eng. 121, 245-253.

Downar, T., Lee, D., Xu Y., Kozlowski, T., Staudenmier, J., 2004. PARCS v2.6 U.S. NRC Core Neutronics Simulator, Theory Manual.

Grundmann, U., Hollstein, F., 1999. A two-dimensional intranodal flux expansion method for hexagonal geometry. Nucl. Sci. Eng. 133, 201-212.

Ivanov, B.D., Ivanov, K.N., Groudev, P., Pavlova, M., Hadjiev, V., 2004. VVER-1000 Coolant Transient Benchmark-Phase 1 (V1000CT-1), vol. 1, Final Specifications (Revision 4), PennState University, Pennsylvania, USA

Ivanov, B.D., Aniel, S., Siltanen, P., Royer, E., Ivanov, K.N., 2006. Impact of crosssection generation procedures on the simulation of the VVER-1000 pump startup experiment in the OECD/DOE/CEA V1000CT benchmark by coupled 3D thermal-hydraulics/neutron kinetics models. Progr. Nucl. Energy 48, 746-763.

Ivanov, B.D., Ivanov, K.N., 2007. VVER-1000 Coolant Transient Benchmark-Phase 1 (V1000CT-1), vol. 3, Summary Results of Exercise 2 on Coupled 3D Kinetics/Core Thermal-hydraulics, PennState University, Pennsylvania, USA.

Jackson, J.W., Todreas, N.E., 1981. COBRA-IIIc/MIT-2: A Computer Program for Steady State and Transient Thermo-Hydraulic Analysis of Rod Bundle Nuclear Fuel Elements, MIT Report.

Jiménez, J., Cuervo, D., Aragonés, J.M., 2007. Multi-scale and Multi-Physics Coupling in COBAYA3, NURETH-12, Pittsburgh, USA

Jiménez, J., Avramova, A., Cuervo, D., Ivanov, K., 2008. Comparative Analysis of Neutronics/Thermal-Hydraulics Multi-scale Coupling for LWR Analysis, PHYSOR-2008, Interlaken, Switzerland.

Knight, M., Hutt, P., Lewis, I., 1995. Comparison of PANTHER nodal solutions in hexagonal-Z geometry. Nucl. Sci. Eng. 121, 254

Kolev, N.P., Petrov, N., Donov, J., Angelova, D., Aniel, S., Royer, E., Ivanov, B., Ivanov, K., Lukanov, E., Dinkov, Y., Popov, D., Nikonov, S., 2006. VVER-1000 Coolant Transient Benchmark (V1000CT-2), vol. II: MSLB Problem - Final Specifications. NEA/NSC/DOC (2006)6.

Kolev, N.P., Petrov, N., Donov, J., 2007a. Specifications of the V1000CT2-EXT2 Multiphysics Benchmark of NURESIM-SP1, Milestone 20b, Work Package T1.4.3.

Kolev, N.P., Petrov, N., Donov, J., Royer, E., 2007b. Comparison of Exercise 2 Results of the OECD VVER-1000 MSLB Benchmark. V1000CT 5th Workshop, Paris, May 7, 2007, in V1000CT 1 and 2 Benchmarks, Cumulative DVD, OECD/NEA.

Lawrence, R.D., 1986. Progress in nodal methods for the solution of the neutron diffusion and transport equations. Prog. Nucl. Energy 17, 271.

Lozano, J.A., Aragonés, J.M., García-Herranz, N., 2007. Development and Performance of the Analytic Nodal Diffusion Solver ANDES in MULTIGROUPS for 3D Rectangular Geometry, M\&C/SNA-2007. Am. Nucl. Soc., Monterey.

Lozano, J.A., García-Herranz, N., Ahnert, C., Aragonés, J.M., 2008a. The analytic nodal diffusion solver ANDES in multigroups for 3D rectangular geometry: development and performance analysis. Ann. Nucl. Energy 35, 2365-2374.

Lozano, J.A., Aragonés, J.M., García-Herranz N., 2008b. Transient Analysis in the 3D Nodal Kinetics and Thermal-Hydraulics ANDES/COBRA coupled system, PHYSOR-2008, Interlaken, Switzerland.

Merino, F., Ahnert, C., Aragonés, J.M., 1993. Development and validation of the 3D PWR core dynamics SIMTRAN code. In: Kusters, H. (Ed.), Mathematical Methods and Supercomputing in Nuclear Applications, vol. 1. ANS-KfK, Karlsruhe, pp. 646-657.

Spasov, I., Jiménez I., Lozano, JA, Herrero, J. 2009 cOBAYA3/COBRAlllc solutions of the V1000CT2-EXT2 Benchmark. Internal Report, Instituto de Fusion Nuclear, Universidad Politécnica de Madrid.

Tomasevic, D.I., Müller, E.Z., 2009. An Extension of the Multigroup Analytic Noda Method (MANM) to Problems in Hexagonal-Z Geometry. In: International Conference on Mathematics, Computational Methods and Reactor Physics (M\&C 2009), Saratoga Springs, NY, May 3-7, 2009, on CD-ROM, American Nuclear Society, LaGrange Park, IL.

Wagner, M.R., 1989. Three dimensional nodal diffusion and transport theory methods for hexagonal-Z geometry. Nucl. Sci. Eng. 103, 377 